\title{
Trombo séptico localizado na topografia de átrio direito em um recém- nascido pré-termo: Relato de caso
}

\author{
Septic thrombus located in the right atrium topography in a preterm newborn: case report \\ Trombo séptico localizado en la topografía de atrio derecho en un recién nacido \\ prematuro: relato de caso
}

Kelly Luisa Cintra ${ }^{1 *}$, Caroline de Abreu Nocera Alves ${ }^{1}$, Felipe Miclos dos Passos ${ }^{1}$, Mayara Araújo da Silva1, Priscilla Lima Martins ${ }^{1}$, Ana Elisa Carvalho Pimenta².

\section{RESUMO}

Detalhamento do caso: Recém-nascido do sexo feminino, idade gestacional de 33 semanas, internada na unidade de terapia intensiva neonatal devido a síndrome da membrana hialina. Evoluiu com quadro de sepse, necessitando de antibioticoterapia e instalação de cateter venoso central em membro superior direito. Após persistência da sepse, foi solicitado ecocardiograma que visualizou imagem de trombo em átrio direito de provável etiologia séptica, pedunculado, móvel, aderido ao septo atrial. Paciente foi submetida a trombólise e evoluiu com melhora clínica e seguimento ambulatorial após alta hospitalar. Discussão: A persistência de quadro sépticos em recém-nascidos leva a suspeita de trombos sépticos, principalmente quando há instalação de cateter venoso central, que é considerado o maior fator de risco. O diagnóstico é feito preferencialmente através do ecocardiograma ou arteriografia contrastada e o tratamento consiste na retirada dos cateteres e terapia com trombolíticos.

Palavras-chave: Recém-nascido, Cateter venoso central, Trombo séptico.

\begin{abstract}
Case detail: Newborn female, gestational age of 33 weeks, admitted to the neonatal intensive care unit due to hyaline membrane syndrome. It evolved with sepsis, necessitating antibiotic therapy and installation of a central venous catheter in the right upper limb. After persistence of sepsis, an echocardiogram was performed, which showed a right atrial thrombus image of probable septic etiology, pedunculated, mobile, adhered to the atrial septum. Patient was submitted to thrombolysis and evolved with clinical improvement and outpatient follow-up after discharge. Discussion: The persistence of septic syndrome in newborns leads to the suspicion of septic thrombi, especially when there is a central venous catheter, which is considered the greatest risk factor. The diagnosis is preferably made through echocardiogram or contrast arteriography and the treatment consists of catheter withdrawal and thrombolytic therapy.
\end{abstract}

Key words: Newborns, Central venous catheters, Septic thrombus.

\section{RESUMEN}

Detalle del caso: Recién nacido del sexo femenino, edad gestacional de 33 semanas, internada en la unidad de terapia intensiva neonatal debido al síndrome de la membrana hialina. Evolucionó con cuadro de sepsis, necesitando de antibioticoterapia e instalación de catéter venoso central en miembro superior derecho. Después de la persistencia de la sepsis, se solicitó ecocardiograma que visualizó imagen de trombo en atrio derecho de probable etiología séptica, pedunculado, móvil, adherido al septo auricular. El paciente fue

${ }^{1}$ Faculdade de Medicina da Universidade de Franca (UNIFRAN), Franca-SP. *E-mail: kellycintra@yahoo.com.br

${ }^{2}$ Fundação Santa Casa de Misericórdia de Franca, Franca-SP. 
sometido a trombólisis y evolucionó con mejoría clínica y seguimiento ambulatorial después del alta hospitalaria. Discusión: La persistencia de cuadro séptico en recién nacidos lleva la sospechosa de trombos sépticos, principalmente cuando hay instalación de catéter venoso central, que es considerado el mayor factor de riesgo. El diagnóstico se realiza preferentemente a través del ecocardiograma o arteriografía contrastada y el tratamiento consiste en la retirada de los catéteres y terapia con trombolíticos.

Palabras clave: Recién nacido, Cateter venoso central, Trombo séptico.

\section{INTRODUÇÃO}

O desenvolvimento de trombos em pacientes recém-nascidos não é muito frequente, mas geralmente ocorre em pacientes que estão internados em unidades de terapia intensiva neonatal (FERRARI $\mathrm{F}$ et al., 2001). Os recém-nascidos com infecções sistêmicas, desidratação, cardiopatias congênitas, asfixia têm mais chances de cursar com complicações trombóticas (WILL A, 2015).

Sabe-se que em recém-nascidos, as concentrações plasmáticas dos fatores de coagulação dependentes da vitamina $\mathrm{K}$ e os fatores de contato (XI, XII, pré-calicreína e cininogênio de alto peso molecular) são $70 \%$ inferiores quando comparados aos dos adultos; entretanto, as concentrações neonatais de fibrinogênio, fator V, fator VIII, fator de Von Willebrand (FVW) e fator XIII chegam a ser 70\% superiores, no recém-nascido prétermo essa diferença é ainda maior, porém atinge níveis equivalentes aos do recém-nascido termo em torno de seis meses após o nascimento (LOPES JRB, et al, 2017).

Nos recém-nascidos com comorbidades, haverá desbalanços desses fatores, podendo apresentar etiologias diferentes que interagem: dano endotelial, redução do fluxo e um estado pró-coagulante, predispondo a sangramentos ou trombogênese (CHAWLA YK e BODH V, 2015). As manifestações clínicas da trombose venosa são variáveis. A maioria dos casos são assintomáticos, sendo descobertos acidentalmente e os sinais dependem da localização do trombo (EDSTROM CS, et al.,2000).

Os fatores de risco compreendem cuidados intensivos, principalmente em recém-nascidos pré-termos, recém-nascidos que necessitam de uso de cateter venoso central, terapia com antibióticos de amplo espectro, nutrição parenteral, uso de hemoderivados, além de sepse, desidratação e peso ao nascer abaixo de 1.250 g (LOPES JRB, et al, 2017).

Aproximadamente $80 \%$ dos eventos tromboembólicos em recém-nascido é secundário a utilização de cateter venoso central. Após poucos dias da implantação dos cateteres, estes aumentam os riscos de eventos adversos graves em recém-nascidos pré-termos, como obstrução valvar com insuficiência cardíaca, pulmonar e/ou tromboembolismo sistêmico, síndrome da veia cava superior ou inferior e oclusão da artéria pulmonar (EDSTROM CS, et al., 2000).

Existem vários possíveis mecanismos pelos quais os cateteres venosos centrais causam trombose incluindo danos às paredes dos vasos, fluxo sanguíneo interrompido, infusão de substâncias como nutrição parenteral total, que danifica as células endoteliais e a presença de materiais trombogênicos nos cateteres (JESUS VC e SECOLI SR, 2007). Quando o trombo atinge a topografia do átrio direito leva a sintomas que incluem insuficiência cardíaca, mau funcionamento do cateter, sepse persistente e aparecimento de um novo sopro cardíaco (ANDREW ME, et al., 2001).

O padrão ouro para o diagnóstico de trombose é a arteriografia. É um exame de alta sensibilidade e menos examinador dependente do que outros métodos de avaliação, porém, a ecocardiografia é a mais utilizada para o diagnóstico visto a grande vantagem de não ser invasiva, não expõe o paciente a radiação, pode ser realizado à beira leito e acompanha a evolução ecocardiográfica do trombo ao longo do tratamento adotado (EDSTROM CS, et al., 2000). Há controvérsias quanto ao tratamento (clínico ou cirúrgico) da trombose intracardíaca em neonatos, pois não existem evidências científicas suficientes que norteiam a melhor conduta até 0 momento. Parâmetros como o tamanho do trombo, mobilidade, se pedunculado são levados em consideração para a escolha do tratamento (YANG JY, et al., 2010). 
Como tratamento clínico a ser instituído a heparina de baixo peso molecular pode ser utilizada com segurança neste grupo etário diminuindo os riscos de sangramento que a anticoagulação oral ou heparina de alto peso molecular poderia ocasionar (ANDREW ME, et al., 2001; EDSTROM CS, et al., 2000).

\section{DETALHAMENTO DO CASO}

Recém-nascido ( $R N$ ), sexo feminino, prematuro moderado (idade gestacional 33 semanas), parto cesárea, membranas íntegras, escore de Apgar 8/9, pesando $1730 \mathrm{~g}$, com perímetro cefálico de $28 \mathrm{~cm}$ e comprimento de $40 \mathrm{~cm}$. Mãe realizou pré-natal, história de adicção ao longo de toda a gestação, apresentou como intercorrência no pré-natal placenta prévia e descolamento prematuro de placenta.

RN foi internada na unidade de terapia intensiva pediátrica (UTIP) da Santa Casa de Misericórdia de Franca logo após o nascimento, dia 05/07/2018, em decorrência de quadro de desconforto respiratório precoce, realizado surfactante exógeno devido a quadro de membrana hialina grau II. Evoluiu satisfatoriamente o padrão respiratório e após dois dias no setor de cuidados intermediários neonatais a paciente iniciou com quadro de distensão abdominal e queda de saturação, evoluindo com sinais inflamatórios sistêmicos tais como taquicardia, taquidispnéia, hipertermia.

Realizado rastreio infeccioso apresentando score hematológico de Rodwell de 2, sendo iniciado oxacilina e amicacina devido a clínica. Apresentou hemocultura com presença de Staphylococcus aureus resistente à meticilina (MRSA), sendo suspenso a antibioticoterapia prescrita anteriormente e iniciado vancomicina além da colocação de cateter venoso central de insersão periférica (PICC) em membro superior direito (MSD). RN manteve quadro séptico e apresentou piora do estado geral, necessitando no dia 21/07/2018, de oxigênio inalatório, expansão volêmica com solução isotônica e concentrado de hemácias. Optado por associar cefepime ao esquema terapêutico vigente e aumentar a dose da vancomicina para cobertura do sistema nervoso central uma vez que não havia sido possível a coleta de líquor.

No dia 24/07/2018 foi optado pela troca do cateter central de inserção percutânea uma vez que a paciente havia dado sinais clínicos de piora, manutenção do quadro séptico mesmo com a terapêutica adequada, surgimento de sopro não havia sido auscultado em nenhum exame físico e sinais de insuficiência cardíaca (Figura 1).

Figura 1- Radiografia de tórax demostrando área cardíaca elevada com sinais de Insuficiência cardíaca.

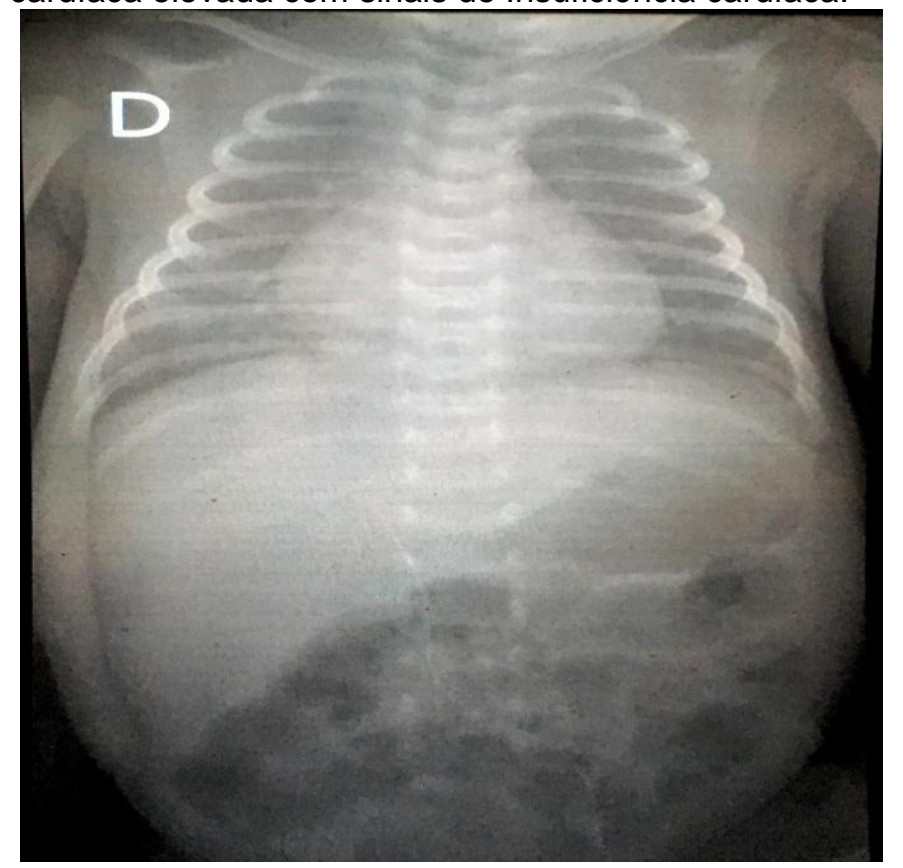

Fonte: Cintra KL, et al; 2018. 
Novo cateter foi puncionado em membro inferior esquerdo e realizado cultura de amostra de ponta de cateter e hemocultura pareada (Figura 2).

Figura 2- Radiografia de tórax com a presença do PICC em MSD. Imagem radiológica antes de iniciar a clínica de insuficiência cardíaca e sepse persistente acima descrita.

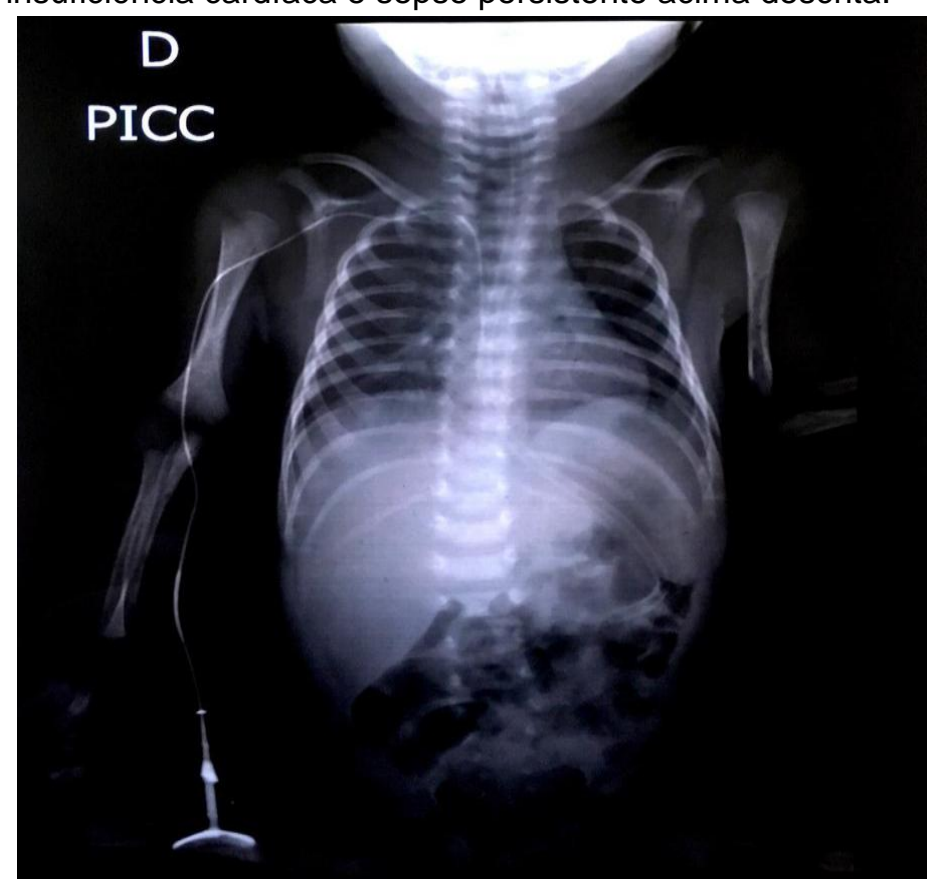

Fonte: Cintra KL, et al; 2018.

Paciente foi readmitida na UTIP no dia 25/07/2018, devido a sepse neonatal tardia por Staphylococcus aureus resistente à meticilina, apnéia e meningite, associado à antibioticoterapia vigente sulfametoxazol $\mathrm{e}$ trimetropim devido a possibilidade de imunossupressão. Realizado ecocardiograma em que foi visualizado imagem de trombo em átrio direito de provável etiologia séptica, pedunculado, móvel, aderido ao septo atrial, com 14x4mm (Figura 3), que se insinuava para dentro da valva tricúspide, sem disfunção valvar). Devido a esse novo diagnóstico, solicitado avaliação da cardiologia pediátrica, sendo iniciado enoxaparina $1,5 \mathrm{mg} / \mathrm{kg} / \mathrm{dose}$ de $12 \mathrm{em} 12$ horas e manutenção da antibioticoterapia (vancomicina e sulfametoxazol+trimetropim), em dieta com sonda orogastrica. Recebeu alta da UTIP no dia 04/08/2018, mantendo acompanhamento e tratamento na unidade de cuidados intermediários neonatais.

Figura 3 - Ecocardiograma com presença de trombo em topografia de átrio direito.

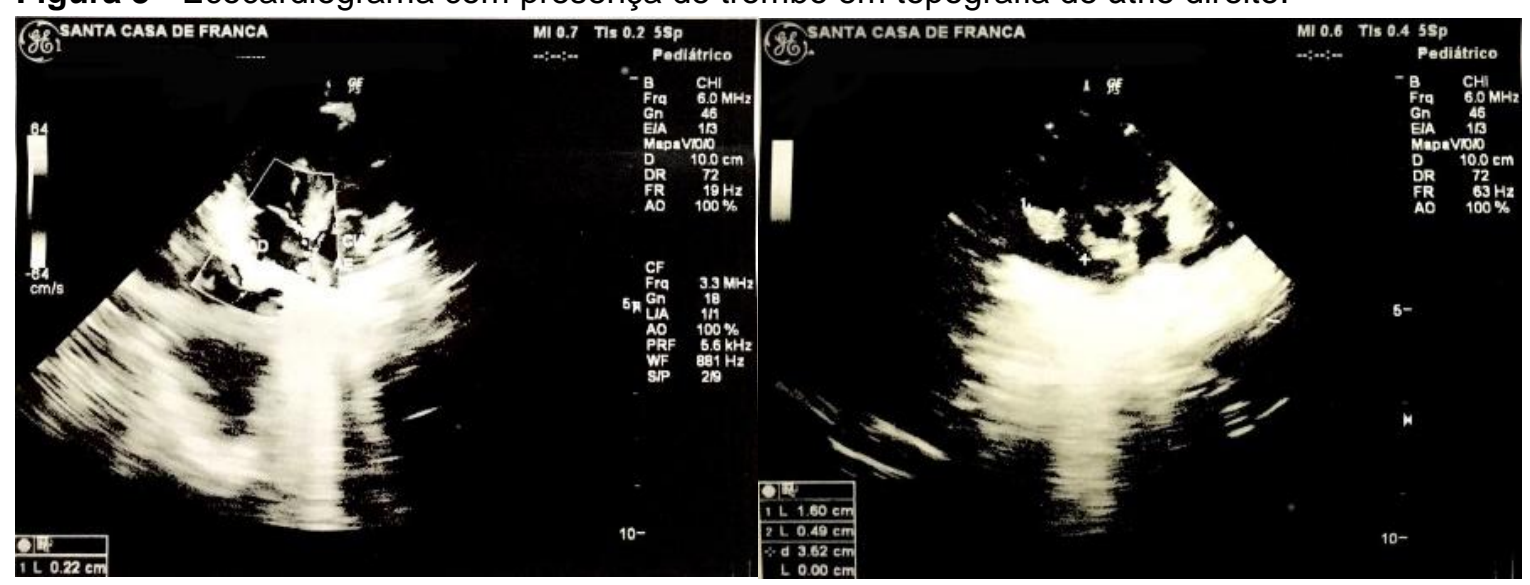

Fonte: Cintra KL, et al; 2018. 
Realizado tratamento com enoxaparina por 8 semanas, sendo acompanhado por exames ecocardiográficos seriados para avaliação da regressão do trombo, ao final evidenciou ausência de trombos, sendo então suspenso a medicação (Figura 4). Durante a terapêutica de anticoagulação com a heparina de baixo peso molecular não foram observados efeitos adversos. Após alta, paciente encontra-se em acompanhamento no ambulatório da criança de alto risco.

Figura 4 - Ecocardiograma após terapêutica de anticoagulação.

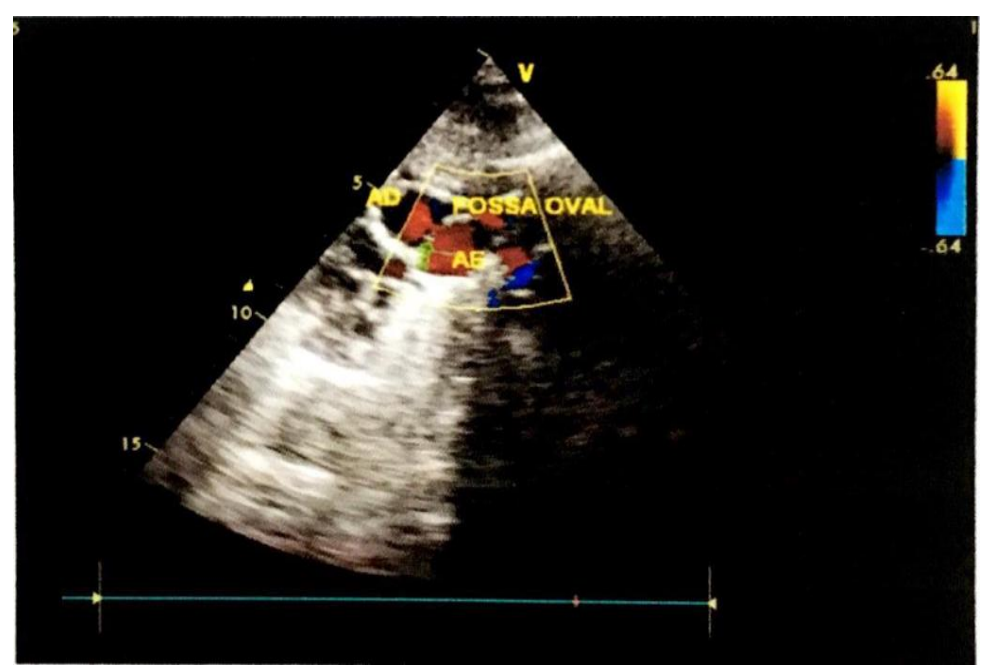

Fonte: Cintra KL, et al; 2018.

\section{DISCUSSÃO}

A incidência de eventos trombóticos neonatais vem aumentando progressivamente ao longo dos últimos anos sendo que a maior incidência é encontrada em pacientes que ocupam leitos de unidades de terapia intensiva neonatal e que necessitam de cateteres venosos centrais, afeta igualmente, ambos os sexos. No estudo prospectivo de Van Omenn, a presença de cateter venoso central como fator de risco para trombose nos recém-nascidos resultou em $94 \%$ dos casos, seguidos por infecções (60\%) e asfixia (13\%) (EDSTROM CS, et al., 2000; VAN OMMEN CH, et al., 2001).

A paciente em questão havia apresentado alguns fatores de risco para eventos trombóticos ao nascer. Dentre estes, listam-se o mau passado obstétrico, prematuridade, necessidade de internação hospitalar por desconforto respiratório e sobretudo a indicação de utilização de PICC.

A trombose venosa apresenta uma incidência de 0,24 por 10.000 internações em unidades de terapia intensiva neonatais, quase todas secundárias aos cateteres venosos centrais, podendo evoluir com desenvolvimento de vegetações intracardíacas, principalmente em átrio direito. A incidência ainda é desconhecida e podem ser secundárias a infecções devido a exposição do lactente à infecção prolongada e à disseminação de êmbolos sépticos, para a região dos pulmões ou obstrução da artéria pulmonar direita (FERRARI F, et al., 2001; ANDREW ME, et al., 2001; TORRES-VALDIVIESO MJ, et al., 2003).

Nos pacientes com esse acometimento são evidenciados sintomas os quais incluem insuficiência cardíaca, mau funcionamento do cateter venoso central, sepse persistente e aparecimento de um novo sopro cardíaco. Após a realização do primeiro ecocardiograma concluiu-se que havia normalidade das câmaras cardíacas, preservação do desempenho sistólico dos ventrículos e a presença do trombo em átrio direito. Logo, o exame excluiu a presença de insuficiência cardíaca por déficit inotrópico e confirmou a presença do trombo em átrio direito que pode levar a uma insuficiência cardíaca por obstrução ao fluxo de entrada no átrio direito e retorno retrógrado para as veias cavas superior e inferior ou ainda o trombo invadir a valva tricúspide ocasionando lesão da valva atrioventricular com consequências prejuízos futuros para a vida da paciente (ANDREW ME, et al., 2001). 
Quanto ao diagnóstico, o ecocardiograma tem a vantagem de não ser invasivo, não expor a radiação e pode ser realizado a beira leito, importante em casos de recém-nascidos pequenos e instáveis, são úteis em quadros suspeitos de acometimento de átrio direito, veia cava ou veia renal. A conduta imediata após a suspeita ou confirmação do caso, é a retirada do cateter e assim iniciada terapêutica adequada de acordo com cada caso (EDSTROM CS, et al., 2000).

Em um estudo sistemático com revisão em neonatos e crianças, trombos atriais de pequeno risco menores que $2 \mathrm{~cm}$, não pedunculados, móveis, podem ser tratados de maneira conservadora. Apesar do contido na literatura, o caso apresentado foi optado por tratamento terapêutico, uma vez que a paciente apresentava um shunt atrial, podendo então considerar o trombo mesmo que pequeno, de alto risco, onde seu deslocamento poderia levar a complicações, como, acidente vascular encefálico, insuficiência de valva tricúspide e até embolia pulmonar (YANG JY, et al., 2010).

Em caso de tratamento medicamentoso, segundo estudos comparativos, a droga ideal é aquela que possibilita a administração por via oral, subcutânea e intravenosa, tenha necessidade de monitoramento mínimo e poucos efeitos colaterais. Os novos anticoagulantes como o inibidor direto da trombina oral (melgatrana) preencheriam todos esses critérios, porém não foram testados em recém-nascidos.

Dentre as medicações disponíveis a HBPM mostra-se superior a outras drogas, pois apresenta farmacocinética mais previsível, monitoramento mínimo, administração subcutânea e menos risco de sangramento e osteopenia se for comparada a heparinas não fracionadas. A HBPM mais utilizada é a enoxaparina que possui uma menor depuração em RN do que em crianças, e necessita de uma menor dose de medicação para atingir níveis adultos de anti-Xa. A medicação de escolha neste caso foi a enoxaparina, por 8 semanas associado a antibioticoterapia endovenoso acompanhada por exames ecocardiográficos seriados para avaliação da regressão do trombo, ao final evidenciou ausência de trombos, sendo então suspenso a medicação (ANDREW ME, et al., 2001).

O principal efeito adverso encontrado em estudos é o sangramento, sendo que em recém-nascidos existem poucos casos relatados, porém no caso relatado a paciente não apresentou nenhuma complicação durante o tratamento (ANDREW ME, et al., 2001).

\section{REFERÊNCIAS}

1. ANDREW ME, et al. Thromboembolic Disease and Antithrombotic Therapy in Newborns. American Society of Hematology, 2001; 359: 358-374.

2. CHAWLA YK, BODH V. Portal vein thrombosis. J ClinExpHepatol. 2015; 5(1):22-40.

3. EDSTROM CS, CHRISTENSEN RD. Evaluation and treatment of thrombosis in the neonatal intensive care unit. Clinics in Perinatology, 2000; 27(3), 623-641

4. FERRARI F, et al. Early intracardiac thrombosis in preterm infants and thrombolysis with recombinant tissue type plasminogen activator. Arch Dis Child Fetal Neonatal, 2001; 85:F66-F72.

5. GREENWAY A, et al. Neonatal thrombosis and its treatment. Blood Ver, 2004; 18: 75-84.

6. JESUS VC, SECOLI SR. Complicações acerca do cateter venoso central de inserção periférica (PICC). Ciência, Cuidado e Saúde. 2007 abril; 6 (2):252-60

7. LOPES JRB, et al. Trombose de veia porta após cateterismo venoso umbilical: revisão da epidemiologia, profilaxia, diagnóstico e tratamento. Rev Med Minas Gerais 2017; 27 (Supl 3): S87-S95

8. TORRES-VALDIVIESO MJ, et al. Successful use of tissue plasminogen activator in catheter-related intracardiac thrombus of a premature infant. J Perinatol, 2003; 20: 91-96.

9. VAN OMMEN CH, et al. Venous thromboembolism in childhood: a prospective two-year registry in the Netherlands. J Pediatr, 2001; 139: 676-681.

10. WILL A. Neonatal haemostasis and the management of neonatal thrombosis. Br J Haematol. 2015 Jan; 169(1):32432.

11. YANG JY, et al. Neonatal and childhood right atrial thrombosis: recognition and a risk-stratified treatment approach. Blood Coagul Fibrinolysis, 2010; 21:301. 